\title{
Usefulness of Endoscopic Hill Grade in Evaluating Children Suspected of Having Gastroesophageal Reflux Disease
}

\author{
In Hyuk Yoo, ${ }^{1}$ Jung Yeon Joo, ${ }^{1}$ and Hye Ran Yang ${ }^{1,2 *}$ \\ ${ }^{1}$ Department of Pediatrics, Seoul National University Bundang Hospital, Seongnam, Gyeonggi-do, Korea and ${ }^{2}$ Department of Pediatrics, Seoul \\ National University College of Medicine, Seoul, Korea
}

\section{Background/Aims}

This study aim to evaluate the relationship between the Hill grade confirmed by esophagogastroduodenoscopy (EGD) and the degree of gastroesophageal reflux (GER) by 24-hour multichannel intraluminal impedance-pH monitoring (MII-pH) in children suspected of having gastroesophageal reflux disease (GERD).

\section{Methods}

A retrospective review of 105 children and adolescents who underwent EGD and MII-pH for the evaluation of GERD from March 2013 to July 2019 was performed. Clinical features and results of EGD and 24-hour MII-pH were collected and statistically analyzed.

\section{Results}

Hill grades 1, 2, 3, and 4 were identified using EGD in 56 (53.3\%), 22 (22.0\%), $16(15.2 \%)$, and 11 (10.5\%) patients, respectively. As the Hill grade increased, the proportion of neurological diseases $(P<0.001)$ and endoscopic erosive esophagitis $(P<0.001)$ increased significantly. The acid exposure index, bolus exposure index, number of reflux episodes, and number of GER reaching proximal extent on MII-pH increased significantly as the endoscopic Hill grade increased (all $P<0.001$ ). Linear regression analysis revealed an increase in the Hill grade by 1 increased the acid exposure index by $2.0 \%$, bolus exposure index by $0.7 \%$, number of reflux episodes by 18.9 episodes, and the number of GER reaching the proximal esophagus increased by 10.5 episodes on average (all $P<0.001)$.

\section{Conclusions}

Hill grade on EGD was associated with GER in children. Estimating the degree of GER by applying Hill grade in the retroflexion view may be useful in practice when evaluating children suspected with GERD.

(J Neurogastroenterol Motil 2021;27:191-197)

Key Words

Child; Endoscopy; Esophageal pH monitoring; Gastroesophageal reflux

Received: February 19, 2020 Revised: May 13, 2020 Accepted: October 24, 2020

() This is an Open Access article distributed under the terms of the Creative Commons Attribution Non-Commercial License (http://creativecommons. org/licenses/by-nc/4.0) which permits unrestricted non-commercial use, distribution, and reproduction in any medium, provided the original work is properly cited.

*Correspondence: Hye Ran Yang, MD, PhD

Department of Pediatrics, Seoul National University Bundang Hospital, Seoul National University, 82, Gumi-ro 173 Beon-gil, Bundang-gu, Seongnam, Gyeonggi-do 13620, Korea

Tel: +82-31-787-7285, Fax: +82-31-787-4054, E-mail: hryang@snubh.org 


\section{Introduction}

Gastroesophageal reflux (GER) is defined as the passage of the gastric contents into the esophagus and is called gastroesophageal reflux disease (GERD) when GER leads to troublesome symptoms and/or complications. ${ }^{1}$ Heartburn, epigastric pain, and regurgitation are well-known typical symptoms of GERD. ${ }^{2}$ However, atypical symptoms such as dysphagia, vomiting, poor weight gain, wheezing, and chronic cough are also known to be associated with GERD in children. ${ }^{1,3,4}$ GERD often produces atypical symptoms or complications such as recurrent pneumonia, esophagitis, esophageal stricture, and malnutrition especially in young children or children with underlying diseases such as neurological impairment. ${ }^{5,6}$ Thus, the diagnosis of GERD is important in pediatric populations when GERD is suspected due to various clinical symptoms.

However, an accurate diagnosis of GERD is often difficult due to the absence of a single gold standard investigation to diagnose GERD in children. ${ }^{1,2}$ For adults with typical reflux symptoms, 1-2week trials of proton pump inhibitors (PPIs) are used to diagnose GERD if symptoms improve with treatment. ${ }^{7}$ However, the most recently published pediatric GER clinical practice guidelines do not recommend a PPI trial unless there are typical symptoms in older children and adolescents. ${ }^{1}$ Therefore, in many cases, the PPI trial cannot be used to diagnose GERD in children. ${ }^{8}$

The 24-hour multichannel intraluminal impedance-pH monitoring (MII-pH) is a new technique that measures all reflux episodes, including acidic, weakly acidic, and alkaline refluxes. ${ }^{9}$ As it is a method to objectively check the degree of reflux, the detection of reflux episodes is more accurate than the conventionally used 24hour $\mathrm{pH}$ monitoring. ${ }^{10}$ Therefore, it is increasingly used as a test for pediatric GERD. ${ }^{11,12}$ However, the MII-pH test is not available in all hospitals and there are some difficulties in applying it to young children.

The gastroesophageal flap valve (GEFV) is a $180^{\circ}$ musculomucosal fold opposite to the lesser curvature of the stomach as viewed with a retroflexed esophagogastroduodenoscopy (EGD). ${ }^{13}$ GEFV, along with the lower esophageal sphincter, the crus of the diaphragm, and the intra-abdominal esophagus, are known to play an important role in blocking retrograde flow from the stomach. ${ }^{14}$ Since Hill et $\mathrm{al}^{13}$ created the Hill grade, a grading system for GEFV in 1996, several studies have reported that the Hill grade was associated with acid reflux events and GERD complications in adults. ${ }^{15-18}$ However, even in adults, there are only a few studies on the association between MII-pH results and the Hill grade. ${ }^{19}$ Fur- thermore, there has been only 1 study on the clinical implications of endoscopic GEFV grading in children; thus, further studies are needed. ${ }^{20}$

Therefore, the aim of this study is to evaluate the relationship between Hill grade, which can be confirmed by EGD, and the degree of reflux through MII-pH in children suspected of having GERD.

\section{Materials and Methods}

The study was carried out at the Seoul National University Bundang Hospital. A retrospective review of 105 children and adolescents from March 1, 2013 to July 31, 2019 was performed under the official approval of the Institutional Review Board of Seoul National University Bundang Hospital (IRB No. B-2002-594-103).

Inclusion criteria were those under 18 years of age who underwent both EGD and MII-pH. Patients were examined for chronic gastrointestinal symptoms including vomiting, abdominal pain, dysphagia, or suspected extraesophageal manifestations of GERD such as recurrent aspiration pneumonia, chronic cough, and faltering growth. Children under 1 year of age were excluded because the GEFV did not develop sufficiently during this period. Patients who had previously undergone fundoplication operations and those without retroflexion views on EGD images were also excluded. The EGD and MII-pH tests were performed within the same hospitalization period.

EGD was performed using a GIF-Q260 or GIF-XP260 scope (Olympus, Tokyo, Japan). Endoscopic images and reports were evaluated by a pediatric gastroenterologist. The grades of GEFV were evaluated using a retroflexed view of EGD as defined by the Hill classification and the results were statistically analyzed along with those of the MII-pH test. The grades of GEFV from grades 1 to 4 are shown in Figure 1. The presence of erosive esophagitis was indicated according to the Los Angeles classification. ${ }^{21}$

Data for MII-pH test were recorded using an ambulatory MII-pH monitoring system (ZepHr; Diversatek Healthcare, Highlands Ranch, CO, USA). We used infant, pediatric, and adolescent catheters according to the patient's age. Each catheter had 7 impedance electrodes and a $\mathrm{pH}$-sensitive electrode. The catheter was introduced transnasally and the $\mathrm{pH}$ sensor was placed above the third vertebral body above the diaphragm confirmed by chest radiography. ${ }^{22}$ Anti-acid suppression therapy had been stopped in all patients at least 3 days before the test. Additionally, the patients were encouraged to maintain their usual activities and diet as much as possible during the test. The MII-pH test was carried out for 


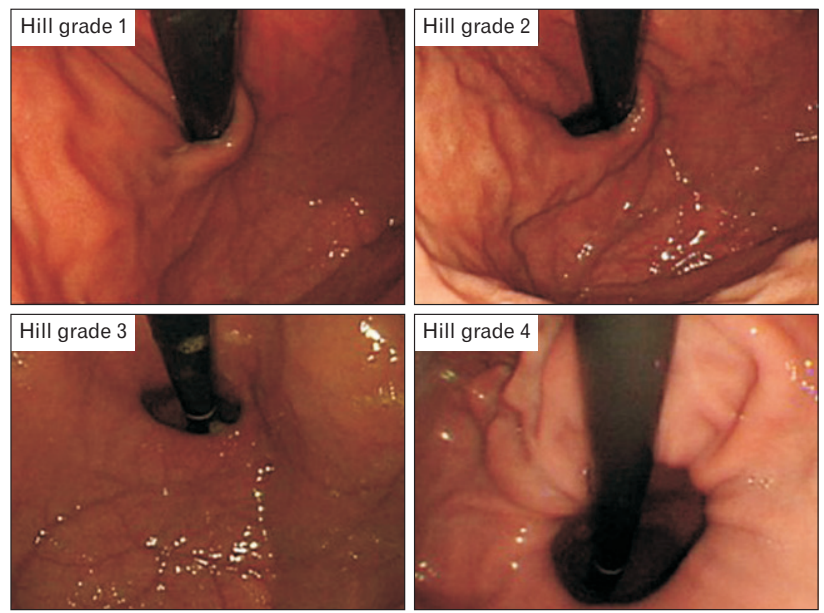

Figure 1. Hill grade on endoscopy. Grade I: presence of a prominent fold of tissue closely approximated to the shaft of the endoscope. Grade II: the fold of tissue is less prominent and there are occasional periods of opening and rapid closing around the endoscope with respiration. Grade III: the fold is barely present, and the endoscope is not tightly gripped by the tissues. Grade IV: there is no fold, and the lumen of the esophagus is open, often allowing the squamous epithelium to be viewed from below. A hiatal hernia is always present.

24 hours and in some patients for at least 20 hours. Consequently, the acid exposure index, bolus exposure index, number of reflux episodes, and number of GER reaching the proximal extent of the esophagus were checked using a software program (Bioview Analysis; Diversatek Healthcare) developed by the manufacturer.

\section{Statistical Methods}

Continuous variables were analyzed using the Kruskal-Wallis test. Linear by linear association was used to compare categorical variables. Linear regression analysis was used to quantify the relationship between the Hill grade and the 24-hour MII-pH test results (acid exposure index, bolus exposure index, number of reflux episodes, and number of GER reaching the proximal extent). For all statistical analyses, a two-sided $P$-value $<0.05$ was considered statistically significant. All statistical analyses were performed using Predictive Analytics SoftWare Statistics (SPSS version 25.0; IBM Corp, Armonk, NY, USA).

\section{Results}

\section{Clinical and Endoscopic Features According to the Hill Grade}

A total of 105 children (57 boys, 48 girls; median age, 6.1 years; interquartile range, 3.3-11.6 years) were recruited in this study. The baseline demographic data and clinical features accord- ing to Hill grade are summarized in Table 1 . A total of 40 patients $(38.1 \%)$ had severe underlying neurologic diseases and 16 patients (15.2\%) had endoscopic erosive esophagitis.

Hill grades $1,2,3$, and 4 were identified in 56 patients $(53.3 \%)$, 22 patients $(22.0 \%), 16$ patients $(15.2 \%)$, and 11 patients $(10.5 \%)$, respectively. As the Hill grade increased, the proportion of neurologic disease $(P=0.002)$ and endoscopic erosive esophagitis $(P<$ $0.001)$ increased significantly.

\section{Comparison of the Findings of Multichannel Intraluminal Impedance-pH Monitoring According to the Hill Grade}

The 24-hour MII-pH test findings of acid exposure index, bolus exposure index, number of reflux episodes, and number of GER reaching proximal extent increased significantly according to the gradual increase in endoscopic Hill grade (all $P<0.001$ ), as shown in Table 2 and Figure 2.

When the Hill grades and MII-pH results were analyzed by linear regression analysis, an increase in Hill grade by 1 was observed to increase the acid exposure index by $2.0 \%(P<0.001)$, the bolus exposure index by $0.7 \%$ ( $P<0.001)$, the number of reflux episodes by 18.9 episodes $(P<0.001)$, and the number of GER reaching the proximal esophagus increased by 10.5 episodes $(P<$ 0.001 ) on average, all of which were statistically significant. 
Table 1. Comparison of Clinical Features According to the Hill Grade Upon Endoscopy

\begin{tabular}{|c|c|c|c|c|c|}
\hline Variable & $\begin{array}{l}\text { Hill grade } 1 \\
(\mathrm{n}=56)\end{array}$ & $\begin{array}{l}\text { Hill grade } 2 \\
\quad(n=22)\end{array}$ & $\begin{array}{l}\text { Hill grade } 3 \\
(\mathrm{n}=16)\end{array}$ & $\begin{array}{l}\text { Hill grade } 4 \\
\quad(\mathrm{n}=11)\end{array}$ & $P$-value ${ }^{\mathrm{a}}$ \\
\hline Age (yr) & $6.0(3.8-10.7)$ & $5.5(2.9-11.9)$ & $6.7(3.2-13.7)$ & $6.6(2.9-12.0)$ & 0.947 \\
\hline Male & $29(51.8)$ & $15(68.2)$ & $5(31.3)$ & $8(72.7)$ & 0.739 \\
\hline Severe neurological disease & $15(26.8)$ & $7(31.8)$ & $11(68.8)$ & $7(63.6)$ & 0.002 \\
\hline Endoscopic erosive esophagitis ${ }^{\mathrm{b}}$ & $3(5.4)$ & $4(18.2)$ & $3(18.8)$ & $6(54.5)$ & $<0.001$ \\
\hline LA grade A & 3 & 4 & & & \\
\hline LA grade B & & & 2 & 3 & \\
\hline LA grade C & & & 1 & 2 & \\
\hline LA grade D & & & & 1 & \\
\hline Hiatal hernia & $1(1.8)$ & $0(0.0)$ & $5(31.3)$ & $11(100.0)$ & $<0.001$ \\
\hline
\end{tabular}

${ }^{a}$ Performed using the Kruskal-Wallis or the Linear by linear association, as indicated.

${ }^{b}$ Los Angeles (LA) classification grade for reflux esophagitis on endoscopy.

Values are presented as median (interquartile range) or $\mathrm{n}(\%)$.

Table 2. Comparison of the Findings of 24-Hour Multichannel Intraluminal Impedance-pH Monitoring Test With the Hill Grade on Endoscopy

\begin{tabular}{|c|c|c|c|c|c|}
\hline Variable & $\begin{array}{l}\text { Hill grade } 1 \\
\qquad(\mathrm{n}=56)\end{array}$ & $\begin{array}{l}\text { Hill grade } 2 \\
\quad(n=22)\end{array}$ & $\begin{array}{l}\text { Hill grade } 3 \\
\quad(n=16)\end{array}$ & $\begin{array}{l}\text { Hill grade } 4 \\
\qquad(\mathrm{n}=11)\end{array}$ & $P$-value ${ }^{a}$ \\
\hline Acid exposure index (\%) & $0.4(0.1-0.9)$ & $1.6(0.2-2.7)$ & $3.6(1.5-7.9)$ & $4.2(3.3-11.5)$ & $<0.001$ \\
\hline Number of pH reflux episodes (episode) & $7.1(1.2-12.8)$ & $16.6(6.0-23.4)$ & $20.9(7.4-37.6)$ & $35.1(20.0-48.4)$ & $<0.001$ \\
\hline Number of $\mathrm{pH}$ reflux episodes $>5 \min$ (episode) & $0.0(0.0-0.0)$ & $1.0(0.0-1.9)$ & $1.6(0.3-3.0)$ & $5.2(0.4-9.8)$ & $<0.001$ \\
\hline Mean acid clearance time (sec) & $26.0(11.8-57.3)$ & $86.0(27.3-166.8)$ & $106.5(83.0-238.8)$ & $190.0(67.0-233.0)$ & $<0.001$ \\
\hline DeMeester score & $1.3(0.9-2.8)$ & $5.2(1.9-10.8)$ & $9.9(3.8-19.1)$ & $13.3(7.7-32.6)$ & $<0.001$ \\
\hline \multicolumn{6}{|l|}{ Number of reflux episodes (episode) } \\
\hline Total & $28.0(14.8-40.0)$ & $45.5(26.0-57.5)$ & $49.5(33.2-79.3)$ & $90.0(46.0-124.0)$ & $<0.001$ \\
\hline Acid & $9.0(3.0-18.8)$ & $20.0(8.5-38.0)$ & $25.5(9.0-42.8)$ & $43.0(33.0-78.0)$ & $<0.001$ \\
\hline Weakly acidic & $14.0(7.0-22.0)$ & $19.0(12.3-35.0)$ & $19.0(6.0-27.8)$ & $20.0(15.0-70.0)$ & 0.048 \\
\hline Weakly alkaline & $0.0(0.0-1.0)$ & $0.0(0.0-1.0)$ & $0.0(0.0-0.8)$ & $0.0(0.0-2.0)$ & 0.720 \\
\hline \multicolumn{6}{|c|}{ Number of gastroesophageal reflux reaching proximal extent (episode) } \\
\hline Total & $12.0(6.3-19.8)$ & $21.5(12.3-39.0)$ & $23.5(14.3-52.2)$ & $33.0(24.0-70.0)$ & $<0.001$ \\
\hline Acid & $5.0(1.0-10.8)$ & $13.5(4.0-26.3)$ & $15.0(6.0-30.0)$ & $25.0(11.0-57.0)$ & $<0.001$ \\
\hline Weakly acidic & $6.0(2.0-10.0)$ & $10.5(4.0-18.0)$ & $7.5(2.0-12.8)$ & $11.0(5.0-24.0)$ & 0.010 \\
\hline Weakly alkaline & $0.0(0.0-0.0)$ & $0.0(0.0-0.3)$ & $0.0(0.0-0.0)$ & $0.0(0.0-1.0)$ & 0.459 \\
\hline Bolus exposure index $(\%)$ & $0.8(0.3-1.1)$ & $1.1(0.8-1.9)$ & $1.6(0.8-2.3)$ & $2.3(1.6-4.4)$ & $<0.001$ \\
\hline Mean bolus clearance time (sec) & $12.0(9.0-15.8)$ & $14.0(10.8-17.3)$ & $13.5(8.5-18.5)$ & $13.0(9.0-18.0)$ & 0.371 \\
\hline
\end{tabular}

${ }^{a}$ Performed with the Kruskal-Wallis test.

Values are presented as median (interquartile range).

\section{Discussion}

The GEFV grading system (eg, Hill grade) is simple, reproducible, and offers useful information when performing endoscopy in patients suspected of having GERD. ${ }^{13}$ Since the Hill grade was established in 1996, several studies have been conducted on the correlation between the Hill grade and GERD symptoms and the results of esophageal manometry or MII-pH in adults. ${ }^{15-17,19} \mathrm{Nev-}$ ertheless, to our knowledge, only 1 study has been conducted on this topic in children to date, demonstrating that abnormal GEFV (Hill grades 3 and 4) was associated with acid reflux. ${ }^{20}$ Thus, further research is required to apply this concept to pediatric practice with more evidence.

In the present study, the relationship between Hill grading on endoscopy and the status of GER on MII-pH were evaluated in children for the first time. In addition to the results of a previous pediatric study, we found that the reflux significantly increased by the 

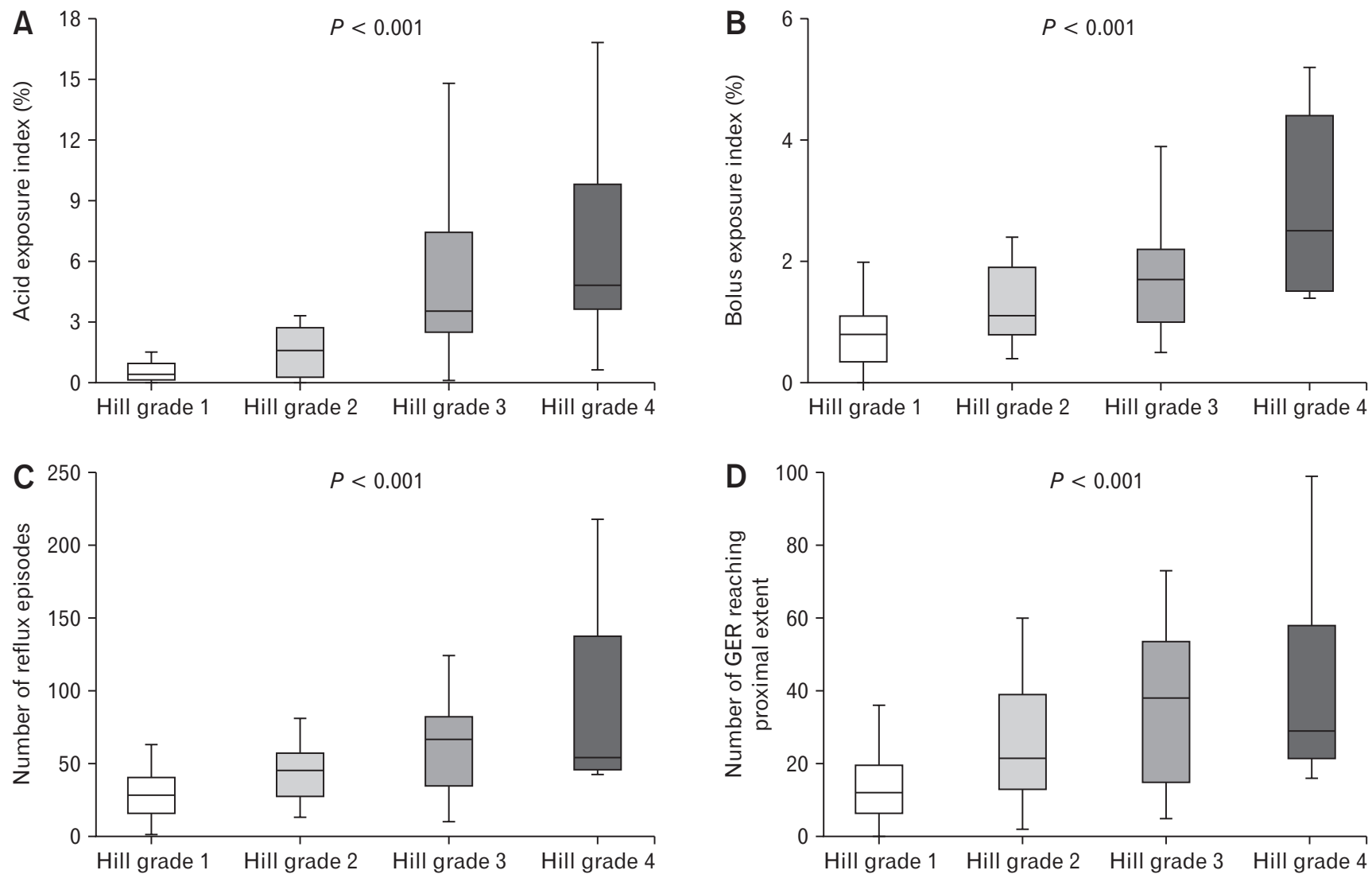

Figure 2. Comparison of the findings of 24-hour multichannel intraluminal impedance-pH monitoring test according to the Hill grade upon endoscopy. (A) Significant positive correlation between Hill grade and acid exposure index. (B) Significant positive correlation between the Hill grade and bolus exposure index. (C) Significant positive correlation between Hill grade and number of reflux episodes. (D) Significant positive correlation between Hill grade and number of gastroesophageal refluxes (GER) reaching the proximal extent. Statistical analysis was performed using the Kruskal-Wallis test.

gradual increase of the Hill grade. Also, the increase in the bolus reflux was statistically significant. These results are similar to those of previous studies on adults, demonstrating the clinical functionality of the Hill grade even in pediatric patients. ${ }^{19}$

As the Hill grade increased, the proportion of endoscopic erosive esophagitis was also significantly observed to increase in our study. This result is similar to that of previous pediatric and adult studies, showing a correlation between the Hill grade and GER. ${ }^{16,17,20}$ The difference from the MII-pH results is that endoscopic erosive esophagitis did not increase significantly in Hill grade 3 , but increased rapidly in Hill grade 4 . This suggests that endoscopic erosive esophagitis is associated with the severe reflux that occurs in Hill grade 4.

In our study, 40 of the 105 study subjects had underlying severe neurological diseases. Underlying neurological diseases varied, including cerebral palsy $(\mathrm{n}=16,40 \%)$, infantile spasm $(\mathrm{n}=2$,
$5 \%)$, Lennox-Gastaut syndrome ( $\mathrm{n}=8,20 \%)$, and brain injury $(\mathrm{n}=8,20 \%)$. Patients with severe neurological disease were significantly higher in Hill grades 3 and 4, and the patients with severe neurological disease were at higher risk for GEFV dysfunction and GERD. This may be explained by the weakening of GEFV caused by reflux in association with many factors such as bed-ridden status, seizures, spasticity, and liquid food feeding via oral or enteral route, which are prevalent in patients with severe neurological disease.

GERD is a common diagnosis in children. When a patient has typical symptoms of GERD such as heartburn, epigastric pain, and regurgitation, a clinical diagnosis may be made through history taking and physical examination. ${ }^{2}$ However, in clinical practice, the diagnosis is not easy because the symptoms are often atypical, and in some cases, further evaluation is needed because the symptoms do not improve easily. In these cases, accurate diagnosis of GERD is important. The pediatric GER clinical practice guidelines pub- 
lished in 2018 recommend that if there is a suspicious alarm sign, the tests are necessary to diagnose the disease, and if not, the EGD and 24-hour MII-pH tests should be performed in stages. ${ }^{1}$

MII-pH is an objective test to determine the degree of GER. ${ }^{9}$ However, there are various limitations in conducting the 24-hour MII-pH test in actual clinical practice. ${ }^{1,12}$ First, there are many hospitals where MII-pH is not available, especially in children. In addition, the test takes a relatively long time (24-hour) with the probe inserted. Since the test is very difficult for a child, the parents of the patient often hesitate to receive the test. Therefore, even if MII-pH is actually required, the test is often canceled or delayed.

EGD in the pediatric population has evolved over the last 30 years with an increasing number of diagnostic and therapeutic applications. ${ }^{23}$ EGD is not an essential test for the diagnosis of GERD, but is useful in detecting the complications of GERD or in diagnosing conditions that may mimic GERD or other gastrointestinal symptoms. ${ }^{1}$ Although EGD is an invasive test compared to other imaging tests, the test time is much shorter and the patient's compliance is significantly better than that of MII-pH. Under these circumstances, a method of indirectly predicting the degree of reflux in patients undergoing EGD for various reasons may provide additional clinical benefits. In addition, it may be beneficial if MII$\mathrm{pH}$ fails or is rejected by patients with suspected GERD symptoms or complications.

The pediatric MII-pH guideline defines MII-pH abnormalities when there are more than 70 reflux episodes in patients aged 1 year or older. ${ }^{24}$ Applying this to the interpretation of the results of our study, it can be said that there may be abnormal reflux in some Hill grade 3 patients and in a large number of Hill grade 4 patients. Therefore, patients with Hill grade 3 or higher on EGD may be considered to have some clinically significant reflux, which may be helpful for the diagnosis and treatment of GERD when combined with clinical symptoms and physical examination findings.

This study has a few limitations as it was performed in a retrospective manner. Although 1 investigator thoroughly reviewed all previous endoscopic images to evaluate the Hill grade, the accuracy may have decreased as the Hill grade was not evaluated at the time of examination in all the study subjects. In addition, the symptom index was not included in the MII-pH results as the study included many patients with neurological diseases who were unable to confirm the exact symptom occurrence each time. Considering that the symptom index is also important for the interpretation of MII-pH results, this may be one of the limitations of our study.

In conclusion, endoscopic Hill grade was associated with GER in pediatric patients. Estimating the degree of GER by Hill grade through EGD can be an assistive aid in evaluating children suspected of having GERD, which can be very useful clinically.

\section{Financial support: None.}

\section{Conflicts of interest: None.}

Author contributions: In Hyuk Yoo: conception and design, and drafting the article; Jung Yeon Joo: analysis and interpretation of data; and Hye Ran Yang: conception and design, and final approval of the version to be published.

\section{References}

1. Rosen R, Vandenplas Y, Singendonk M, et al. Pediatric gastroesophageal reflux clinical practice guidelines: joint recommendations of the North American Society for Pediatric Gastroenterology, Hepatology, and Nutrition and the European Society for Pediatric Gastroenterology, Hepatology, and Nutrition. J Pediatr Gastroenterol Nutr 2018;66:516-554.

2. Sherman PM, Hassall E, Fagundes-Neto U, et al. A global, evidencebased consensus on the definition of gastroesophageal reflux disease in the pediatric population. Am J Gastroenterol 2009;104:1278-1295.

3. Vandenplas Y, Rudolph CD, Di Lorenzo C, et al. Pediatric gastroesophageal reflux clinical practice guidelines: joint recommendations of the North American Society for Pediatric Gastroenterology, Hepatology, and Nutrition (NASPGHAN) and the European Society for Pediatric Gastroenterology, Hepatology, and Nutrition (ESPGHAN). J Pediatr Gastroenterol Nutr 2009;49:498-547.

4. Liu XL, Wong KK. Gastroesophageal reflux disease in children. Hong Kong Med J 2012;18:421-428.

5. Vandenplas Y, Hassall E. Mechanisms of gastroesophageal reflux and gastroesophageal reflux disease. J Pediatr Gastroenterol Nutr 2002;35:119-136.

6. Hassall E, Kerr W, El-Serag HB. Characteristics of children receiving proton pump inhibitors continuously for up to 11 years duration. J Pediatr 2007;150:262-267, e1.

7. Bautista J, Fullerton H, Briseno M, Cui H, Fass R. The effect of an empirical trial of high-dose lansoprazole on symptom response of patients with non-cardiac chest pain--a randomized, double-blind, placebocontrolled, crossover trial. Aliment Pharmacol Ther 2004;19:1123-1130.

8. Yang J, Lee J, Lee $\mathrm{H}$, et al. Is the diagnostic trial with proton pump inhibitors reasonable for school age children with gastroesophageal reflux symptoms? Pediatr Gastroenterol Hepatol Nutr 2019;22:511-517.

9. Sifrim D, Castell D, Dent J, Kahrilas PJ. Gastro-oesophageal reflux monitoring: review and consensus report on detection and definitions of acid, non-acid, and gas reflux. Gut 2004;53:1024-1031.

10. Safe M, Cho J, Krishnan U. Combined multichannel intraluminal impedance and $\mathrm{pH}$ measurement in detecting gastroesophageal reflux disease in children. J Pediatr Gastroenterol Nutr 2016;63:e98-e106.

11. Rosen R, Hart K, Nurko S. Does reflux monitoring with multichannel intraluminal impedance change clinical decision making? J Pediatr Gas- 
troenterol Nutr 2011;52:404-407.

12. Shin MS. Esophageal $\mathrm{pH}$ and combined impedance-pH monitoring in children. Pediatr Gastroenterol Hepatol Nutr 2014;17:13-22.

13. Hill LD, Kozarek RA, Kraemer SJ, et al. The gastroesophageal flap valve: in vitro and in vivo observations. Gastrointest Endosc 1996;44:541-547.

14. Gordon C, Kang JY, Neild PJ, Maxwell JD. The role of the hiatus hernia in gastro-oesophageal reflux disease. Aliment Pharmacol Ther 2004;20:719-732.

15. Hansdotter I, Björ O, Andreasson A, et al. Hill classification is superior to the axial length of a hiatal hernia for assessment of the mechanical anti-reflux barrier at the gastroesophageal junction. Endosc Int Open 2016;4:E311-E317.

16. Xie C, Li Y, Zhang N, Xiong L, Chen M, Xiao Y. Gastroesophageal flap valve reflected EGJ morphology and correlated to acid reflux. BMC Gastroenterol 2017;17:118.

17. Oberg S, Peters JH, DeMeester TR, et al. Endoscopic grading of the gastroesophageal valve in patients with symptoms of gastroesophageal reflux disease (GERD). Surg Endosc 1999;13:1184-1188.

18. Quach DT, Nguyen TT, Hiyama T. Abnormal gastroesophageal flap valve is associated with high gastresophageal reflux disease questionnaire score and the severity of gastroesophageal reflux disease in Vietnamese patients with upper gastrointestinal symptoms. J Neurogastroenterol Motil 2018;24:226-232.
19. Koch OO, Spaun G, Antoniou SA, et al. Endoscopic grading of the gastroesophageal flap valve is correlated with reflux activity and can predict the size of the esophageal hiatus in patients with gastroesophageal reflux disease. Surg Endosc 2013;27:4590-4595.

20. Chang KC, Wu JF, Hsu WC, Lin BR, Chen HL, Ni YH. Impacts of endoscopic gastroesophageal flap valve grading on pediatric gastroesophageal reflux disease. PLoS One 2014;9:e107954.

21. Lundell LR, Dent J, Bennett JR, et al. Endoscopic assessment of oesophagitis: clinical and functional correlates and further validation of the Los Angeles classification. Gut 1999;45:172-180.

22. Quitadamo P, Tambucci R, Mancini V, et al. Esophageal $\mathrm{pH}$-impedance monitoring in children: position paper on indications, methodology and interpretation by the SIGENP working group. Dig Liver Dis 2019;51:1522-1536

23. Tringali A, Thomson M, Dumonceau JM, et al. Pediatric gastrointestinal endoscopy: European Society of Gastrointestinal Endoscopy (ESGE) and European Society for Paediatric Gastroenterology Hepatology and Nutrition (ESPGHAN) Guideline Executive summary. Endoscopy 2017;49:83-91.

24. Pilic D, Froölich T, Nöh F, et al. Detection of gastroesophageal reflux in children using combined multichannel intraluminal impedance and $\mathrm{pH}$ measurement: data from the German Pediatric Impedance Group. J Pediatr 2011;158:650-654, e1. 